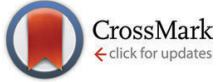

Cite this: Phys. Chem. Chem. Phys. 2016, 18, 12252

Received 23rd December 2015, Accepted 29th March 2016

DOI: $10.1039 / c 5 c p 07936 e$

www.rsc.org/pccp

\title{
Vibrational signatures of the water behaviour upon confinement in nanoporous hydrogels
}

\author{
B. Rossi, ${ }^{a b}$ V. Venuti, ${ }^{c}$ A. Mele, ${ }^{d}$ C. Punta, ${ }^{d}$ L. Melone, ${ }^{\text {de }}$ F. D'Amico, ${ }^{a}$ A. Gessini, ${ }^{a}$ \\ V. Crupi, ${ }^{b}$ D. Majolino, ${ }^{b}$ F. Trotta $^{f}$ and C. Masciovecchio ${ }^{a}$
}

\begin{abstract}
The fundamental question of how the reorganization of the hydrogen-bond (HB) network of water is influenced by the combination of nano-confinement and hydrophobic/hydrophilic solvation effects is addressed here using a spectroscopic study of water absorbed in a model, $\mathrm{pH}$-sensitive polysaccharide hydrogel. The effects of temperature, hydration level and $\mathrm{pH}$ on the vibrational dynamics associated with the water molecules and the polymer skeleton are disentangled and analysed by a complementary and combined use of UV-Raman scattering and IR spectroscopy. The experimental data give evidence that the solvation effects in the hydrogel matrix are essentially dominated by the hydration of more hydrophobic parts of the polymer network, while the effect of $\mathrm{pH}$ on the $\mathrm{HB}$ reorganization of confined water molecules is found to be similar to that induced by cooling of the system. A tentative explanation of these results has been provided in terms of interplay between different kinds of interactions, i.e. hydrophobic vs. hydrophilic.
\end{abstract}

\section{Introduction}

The behaviour of water in the proximity of hydrophobic and hydrophilic groups of a molecule plays a fundamental role in determining several bio-relevant mechanisms such as solvation of solutes, aggregation processes, gelation phenomena and selfassembly of macromolecules. It is well established from many years of research that both the structure and the dynamics of water molecules are strongly modified by the presence of surfaces and molecular chemical sites.

Concerning the first issue, it was experimentally demonstrated that the structural and dynamic features of confined water are different from those exhibited by bulk water. ${ }^{1-8}$ The presence of surfaces induces both a change in the rearrangement of the hydrogen-bond ( $\mathrm{HB}$ ) network of water molecules and modifications of the water molecular motion. ${ }^{1,9}$ Different properties of confined water, especially within nanoscale spaces,

\footnotetext{
${ }^{a}$ Elettra - Sincrotrone Trieste, Strada Statale $14 \mathrm{~km}$ 163.5, Area Science Park, 34149 Trieste, Italy. E-mail: barbara.rossi@elettra.eu

${ }^{b}$ Department of Physics University of Trento and INSTM Local Unit, via Sommarive 14, 38123 Povo, Trento, Italy

${ }^{c}$ Department of Physics and Earth Sciences, University of Messina, Viale Ferdinando Stagno D'Alcontres 31, 98166 Messina, Italy

${ }^{d}$ Department of Chemistry, Materials and Chemical Engineering "G. Natta", Politecnico di Milano and INSTM local unit, Piazza L. da Vinci 32, 20133, Milano, Italy

${ }^{e}$ Università degli Studi e-Campus, Via Isimbardi 10, 22060 Novedrate, Como, Italy

${ }^{f}$ Department of Chemistry, University of Torino, Via Pietro Giuria 7, 10125 Torino, Italy
}

depend on the chemical characteristics of the cavity surface, the confinement dimensions, and temperature and pressure conditions. ${ }^{10-12}$

Concerning the behaviour of water around chemical sites, experimental and numerical studies have found that water molecules undergo cooperative restructuring processes of their HB network in the hydration layers surrounding hydrophobic and hydrophilic portions of biomacromolecules. ${ }^{13-15}$ The influence of small and large molecules on the surrounding solvent molecules has been described in terms of both destructuring/restructuring effects on the whole HB assembly of water molecules ${ }^{16}$ and change in the local dynamics of the solvent at different chemical sites. ${ }^{17-20}$ Recently, we used oligosaccharide based hydrogels as model systems to study the effect on the HB dynamics of water ${ }^{21-24}$ of two factors, i.e. (i) the nano-scale confinement of water and (ii) the presence of hydrophobic/hydrophilic portions at the interface with the solvent. These systems consisted of a nanoporous hydrogel matrix obtained after polymerization and swelling of $\beta$-cyclodextrin, affording a class of cross-linked polymers referred to as cyclodextrin nanosponges (NSs). ${ }^{25}$ The polymerization of cyclodextrins (CDs) is generally carried out with suitable polyfunctional cross-linking (CL) agents and leads to a three-dimensional covalent network characterized by the presence of the hydrophobic cavity of the CD units and more hydrophilic pores of the cross-linked polymer. ${ }^{26}$ The polymer matrix of NSs has shown to be able to retain large fractions of water within its structure, affording homogeneous gel phases. ${ }^{27}$ Besides their potential practical applications as nano-carriers of bioactive agents, ${ }^{28,29}$ the study of the behaviour of water entrapped in NS hydrogels 
can provide new fundamental insights into the water-water and water-polymer interactions in soft confining systems. This is due to the simultaneous presence of hydrogen bond donor/acceptor groups in the polymer skeleton, as well as due to the presence of nano-spaces where water can be confined..$^{21-23}$ The versatility of NS polymers allows one to tune the hydrophilicity/hydrophobicity balance of the polymer groups by acting on some parameters during the synthetic procedure. ${ }^{30-34}$ Moreover, it has been recently demonstrated that the water-retaining ability of NS hydrogels is $\mathrm{pH}$ sensitive. $^{24,26}$ These findings prompted us to use NS hydrogels as soft confining media for investigating the HB dynamics of water upon confinement.

In the liquid phase, water develops a complex network of HB links. The network is characterized by the presence of ordered regions, i.e. tetrahedral $\mathrm{H}$-bonded structures, and a large amount of "closed structures", i.e. regions of defective hydrogen bonds. ${ }^{35}$ This intricate intermolecular network is constantly rearranging (breaking and reforming) depending on the temperature conditions and the perturbation induced by the presence of hydrophobic/hydrophilic chemical groups. Vibrational spectroscopy is a powerful tool for probing the three-dimensional network of hydrogen bonds in liquid water. The vibrational modes of $\mathrm{H}_{2} \mathrm{O}$ are sensitive to the levels of connectivity of the solvent molecules with their neighbours. ${ }^{6-39}$ IR and Raman spectroscopy can be conveniently employed for the quantitative evaluation of the extent of perturbation on water organization due to the combination of confinement effects ${ }^{3,4,6-8}$ and interactions with hydrophobic/ hydrophilic portions of the confining polymeric matrix. ${ }^{15-17,19}$ In the past few years, it was shown that the combination of UV-Raman scattering experiments and FT-IR spectroscopy is a simple strategy to separate the spectral response arising from the polymer matrix and the water molecules. ${ }^{21-24}$ The separate analysis of the vibrational dynamics associated with confined water molecules and the polymer skeleton thus provides new insights into the interplay of different types of HB interactions that coexist in hydrogel phases. ${ }^{21,24}$

In this paper, we focus on the vibrational signatures of the reorganization of the $\mathrm{HB}$ network of water upon confinement in the nano-sized cavities of the polymeric matrix whose surface present both hydrophobic and hydrophilic sites. To this aim, a recently developed $\mathrm{pH}$-sensitive $\mathrm{NS}$ hydrogel ${ }^{24}$ employed as a water confining matrix is used. Such systems show different swelling properties depending on the $\mathrm{pH}$ of the hydrating starting solution. The spectral modifications of intra- and inter-molecular vibrations associated with the $\mathrm{HB}$ network of $\mathrm{H}_{2} \mathrm{O}$ and specific hydrophobic/hydrophilic groups of the polymer skeleton are studied as a function of temperature, hydration level and $\mathrm{pH}$. Particular attention is devoted to the frequency and intensity evolution of the bending mode of $\mathrm{H}_{2} \mathrm{O}$ that is well detected in IR spectra. ${ }^{40,41}$ The structural properties of liquid water can be reliably described by the analysis of the spectral modifications of the bending mode of $\mathrm{H}_{2} \mathrm{O}$ with respect to the usual analysis performed on the $\mathrm{OH}$ stretching profile of $\mathrm{H}_{2} \mathrm{O}$. Although a nonunique behaviour has been found for the $\mathrm{HOH}$ bending mode of water upon confinement, ${ }^{3,4,6,8}$ the comparison of UV-Raman and FTIR-ATR spectra of the NS hydrogel, here presented and discussed, provides information on the behaviour of water as a function of the size of the confining cavities in a model hydrogel. These results are in turn interpreted in the light of the fundamental question of how much HB water dynamics is affected by confinement effects rather than by the presence of hydrophobic/hydrophilic portions at the solvent interface.

\section{Experimental}

\section{A Preparation of nanosponge hydrogels}

$\beta$-Cyclodextrin nanosponge polymers were prepared by reacting $\beta$-cyclodextrin ( $\beta$-CD), dissolved in anhydrous DMSO and in the presence of anhydrous $\mathrm{Et}_{3} \mathrm{~N}$, with the cross-linking agent pyromellitic anhydride (PMA) at room temperature for 3 hours under intense stirring. The cross-linker PMA was added at 1:4 molecular ratios with respect to the monomer $\mathrm{CD}$. Thus, the corresponding polymers can be referred to as $\beta$-CDPMA14, where the digits refer to the $\beta$-CD/PMA molar ratio. The obtained polymer was crushed in a mortar and purified by washing with $0.2 \mathrm{M} \mathrm{HCl}(\mathrm{aq})$ (3 times) and deionized water (5 times), and finally dried under vacuum affording a homogeneous powder. Subsequently, $\beta$-CDPMA14 hydrogels were prepared by adding suitable amounts of double-distilled water (Sigma) to the dry polymer in order to obtain the desired level of hydration $h$ (where $h$ is defined as water/NS weight ratio). Suitable amounts of anhydrous $\mathrm{Na}_{2} \mathrm{CO}_{3}$ were added to the hydrating water solution $(5,10,15,20$ and $25 \% \mathrm{w} / \mathrm{w})$ achieving the final $\mathrm{pH}$ of 8.5, 8.9, 9.2, 9.7 and 10.1, respectively, in the gel phase. After this procedure, a perfectly homogeneous and transparent gel without any visible phase separation or solid particles was obtained in about one hour time.

For FTIR-ATR measurements an additional set of nanosponge hydrogel samples was prepared by hydration of the dry polymer $\beta$-CDPMA14 with deuterated water $\mathrm{D}_{2} \mathrm{O}$, following the same procedure described above. Also in this case the final $\mathrm{pH}$ of the gel phase was adjusted by adding suitable amounts of $\mathrm{Na}_{2} \mathrm{CO}_{3}(5,10,15,20$ and $25 \% \mathrm{w} / \mathrm{w})$ to $\mathrm{D}_{2} \mathrm{O}$ used for the hydration.

All the gel samples were freshly prepared and used for IR and Raman measurements.

\section{B FTIR-ATR absorption measurements}

FTIR-ATR measurements were performed in the temperature range of $250-350 \mathrm{~K}$ on nanosponge hydrogels prepared as described in the previous section. The IR spectra were collected in the $400-4000 \mathrm{~cm}^{-1}$ wavenumber range on a Bomem DA8 Fourier transform spectrometer, operating with a Globar source, in combination with a $\mathrm{KBr}$ beamsplitter and a DTGS/ $\mathrm{KBr}$ detector. The samples were contained in the Golden Gate diamond ATR system, just based on the ATR technique. The spectra were recorded in a dry atmosphere, in order to avoid dirt contributions, with a resolution of $4 \mathrm{~cm}^{-1}$, automatically adding 100 repetitive scans in order to obtain a good signal-tonoise ratio and high reproducibility. All the IR spectra were normalized for taking into account the effective number of absorbers. 
No mathematical correction (e.g., smoothing) was done, and spectroscopic manipulations such as baseline adjustment and normalization were performed using the Spectracalc software package GRAMS (Galactic Industries, Salem, NH, USA).

\section{UV-Raman scattering measurements}

UV-Raman scattering measurements were performed at the BL10.2-IUVS beamline at the Elettra Synchrotron Laboratory in Trieste by exploiting the experimental set-up described in ref. 42. The Raman spectra were excited at $266 \mathrm{~nm}$ and collected in a back-scattered geometry by using a triple stage spectrometer (Trivista, Princeton Instruments). The experimental resolution was set to $5 \mathrm{~cm}^{-1}$ in order to ensure enough resolving power and count-rate. To minimize potential photodecomposition of the gels resulting from UV radiation exposure, the sample cell was subjected to slow continuous spinning during the running of measurements in order to vary the illuminated sample volume through the exciting radiation beam. Polarized parallel $\left(I_{\mathrm{VV}}\right)$ and perpendicular $\left(I_{\mathrm{HV}}\right)$ Raman spectra were acquired at room temperature. The isotropic Raman intensity has been obtained according to the relation: $I_{\mathrm{ISO}}=I_{\mathrm{VV}}-\frac{4}{3} I_{\mathrm{HV}}$.

\section{Results and discussion}

\section{A Hydration-effect on hydrophobic/hydrophilic groups of the hydrogel matrix}

In Fig. 1(a) the isotropic Raman signals obtained for $\beta$-CDPMA14 hydrogels at different hydration levels $h$ are shown in the wavenumber region between 1450 and $1800 \mathrm{~cm}^{-1}$.

As largely discussed in previous studies, ${ }^{21,22,24}$ the isotropic Raman spectrum of the NS hydrogel in this wavenumber range is dominated by the spectral signals arising from the polymer matrix.

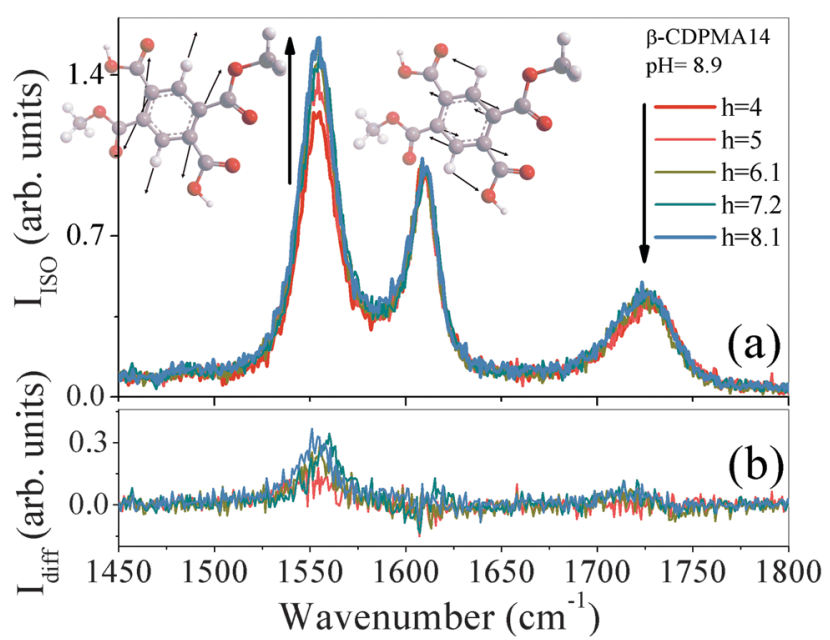

Fig. 1 (a) Hydration-evolution of isotropic Raman profiles for the $\beta$-CDPMA14 nanosponge hydrated with $\mathrm{H}_{2} \mathrm{O}+\mathrm{Na}_{2} \mathrm{CO}_{3} 10 \% \mathrm{w} / \mathrm{w}$ $(\mathrm{pH}=8.9)$. The sketches reported in the graph illustrate the vibrational modes associated with the Raman signals $\nu(\mathrm{C}=\mathrm{C})_{1}$ and $\nu(\mathrm{C}=\mathrm{C})_{2}$. (b) Difference spectral intensities $/_{\text {diff }}$ obtained as described in the text at various hydration values $h$.
Then, the analysis of the frequency, shape and intensity modifications of Raman peaks reported in Fig. 1(a) gives information mainly on the molecular perturbation occurring on specific chemical groups of the polymer skeleton when surrounded by water molecules.

The two peaks at $1554 \mathrm{~cm}^{-1}$ and $1610 \mathrm{~cm}^{-1}$ in the spectra shown in Fig. 1(a) have been associated with Raman active modes involving the aromatic moiety of PMA units of the nanosponge polymer network, ${ }^{21,22,24} \nu(\mathrm{C}=\mathrm{C})_{1}$ and $\nu(\mathrm{C}=\mathrm{C})_{2}$, respectively. More specifically, the $\nu(\mathrm{C}=\mathrm{C})_{1}$ vibrational mode has been assigned to the ring breathing motion of PMA, while $\nu(\mathrm{C}=\mathrm{C})_{2}$ is assigned to the combination of stretching vibrations of the aromatic $\mathrm{C}=\mathrm{C}$ bonds and bending of the $\mathrm{C}-\mathrm{H}$ groups on the PMA unit, ${ }^{21,24}$ as illustrated in the sketches in Fig. 1(a). Conversely, the broad asymmetric band centred at about $1730 \mathrm{~cm}^{-1}$ is assigned to the stretching vibrations of the $\mathrm{C}=\mathrm{O}$ groups of the PMA residues and it is generally indicated as $\nu(\mathrm{C}=\mathrm{O}) \cdot{ }^{34}$ As expected, in the UV-Raman spectra of Fig. 1(a) the intensity of the peaks corresponding to $\nu(\mathrm{C}=\mathrm{C})_{1}$ and $\nu(\mathrm{C}=\mathrm{C})_{2}$ is significantly enhanced with respect to the vibrational features observed in the Raman spectra excited with visible light, ${ }^{34}$ due to the UV resonance effects. ${ }^{21,24}$ This condition provides us with greater sensitivity to the spectral modifications occurring in the vibrational signals selectively associated with the hydrophobic aromatic moiety of the NS polymer network.

The isotropic spectra reported in Fig. 1(a) point out the marked enhancement of the intensity of the mode $\nu(\mathrm{C}=\mathrm{C})_{1}$, as a consequence of the increase of the water content in NS hydrogels. At the same time, no significant spectral modifications can be detected for the Raman bands associated with $\nu(\mathrm{C}=\mathrm{C})_{2}$ and $\nu(\mathrm{C}=\mathrm{O})$ upon increasing $h$. These experimental findings are better highlighted by introducing the difference spectral intensities $I_{\text {diff }}$ (Fig. 1(b)) that have been calculated as:

$$
I_{\text {diff }}(h)=I(h)-I(h=4)
$$

where $I(h)$ is the isotropic Raman spectrum of the NS polymer hydrated at hydration level $h$ and $I(h=4)$ is the curve collected on the NS hydrated at $h=4$, which corresponds to the lowest value of hydration measured.

The hydration-dependence observed for the mode $\nu(\mathrm{C}=\mathrm{C})_{1}$ in the UV Raman spectra of NS hydrogels is quite expected, on the basis of previous results obtained for similar NS hydrogels. ${ }^{21}$ It has been explained in terms of establishment of a $\mathrm{HB}$ of type $\mathrm{C}-\mathrm{H} \cdot \mathrm{O}-\mathrm{O}$ between the activated $\mathrm{CH}$ groups of the aromatic ring of PMA and the water molecules confined in the pores of the hydrogel matrix and surrounding the functional groups of NS. The remarkable sensitivity to the hydration level exhibited by the mode $\nu(\mathrm{C}=\mathrm{C})_{1}$ compared to $\nu(\mathrm{C}=\mathrm{C})_{2}$ supports the directional character of the $\mathrm{C}-\mathrm{H} \cdots \mathrm{O}-\mathrm{H}$ interactions, consistent with what is expected from a genuine hydrogen bond.

In order to gain more quantitative indications about how the intensity of Raman mode $\nu(\mathrm{C}=\mathrm{C})_{1}$ depends on the hydration level $h$, the isotropic Raman spectra have been fitted by using a combination of Kubo-Anderson functions, as described in detail in ref. 21 and 24. In Fig. 2(a), an example of best-fitting results is shown for the experimental curves corresponding to 


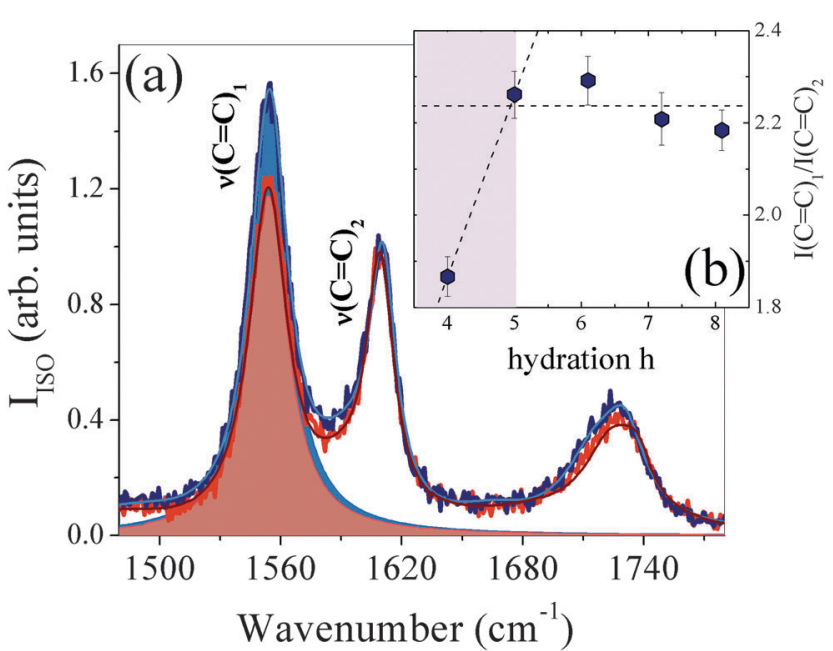

Fig. 2 (a) Experimental isotropic Raman spectra of the $\beta$-CDPMA14 hydrogel hydrated with $\mathrm{H}_{2} \mathrm{O}+\mathrm{Na}_{2} \mathrm{CO}_{3} 10 \% \mathrm{w} / \mathrm{w}$ at $h=4$ (orange line) and $h=8$ (blue line), reported together with the best fits (wine and cyan lines) obtained for the two spectra. The stretching modes $\nu(\mathrm{C}=\mathrm{C})_{1}$ are shown as pink and cyan coloured area, respectively, for the two experimental profiles. (b) Hydration-dependence of the $I(C=C)_{1} / I(C=C)_{2}$ area ratio estimated for the $\beta$-CDPMA14 hydrogel. Dotted lines are guide to the eye to highlight the $h$-evolution.

the NS polymer hydrated at $h=4$ (orange line) and $h=8$ (blue line). The spectral components reproducing the modes $\nu(\mathrm{C}=\mathrm{C})_{1}$ for the two experimental profiles reported in Fig. 2(a) are evidenced as coloured areas. As mentioned above, the intensity of the vibration $\nu(\mathrm{C}=\mathrm{C})_{1}$ can be used as a spectroscopic descriptor of the perturbation induced by water molecules on the vibrational dynamics of the $\mathrm{CH}$ groups of PMA in the NS polymer network. ${ }^{21}$

Fig. 2(b) shows the hydration-evolution of the intensity of mode $\nu(\mathrm{C}=\mathrm{C})_{1}$ normalized to the intensity of $\nu(\mathrm{C}=\mathrm{C})_{2}$, i.e. the peak area ratio $I(\mathrm{C}=\mathrm{C})_{1} / I(\mathrm{C}=\mathrm{C})_{2}$ estimated by the fitting procedure of experimental spectra. The plot of Fig. 2(b) gives evidence that the $\mathrm{pH}$-sensitive NS hydrogels considered in this work exhibit, upon increase of hydration of the polymer, a behaviour similar to that found for other PMA-based nanosponge hydrogels. ${ }^{21}$ In fact, for $h>5$, the ratio $I(C=C)_{1} / I(C=C)_{2}$ becomes practically hydration-independent, thus suggesting that the dynamic perturbation induced on $\mathrm{C}-\mathrm{H}$ groups of PMA by water tends to vanish at higher hydration levels.

Different from what was observed in the case of non-pH sensitive $\mathrm{NSs}^{21}$ the intensity of the mode $\nu(\mathrm{C}=\mathrm{O})$ associated with the carbonyl groups present in the polymer network does not change as a function of the water content of hydrogels. This finding further supports the conclusion that in the case of pH-sensitive NS hydrogels the solvation effects are essentially dominated by the interactions established between the $\mathrm{CH}$ groups located in the hydrophobic part of the polymer (the PMA connector) and the surrounding water molecules. ${ }^{24}$

Based on the results illustrated in Fig. 2(b), a fixed hydration value of $h=4$ has been chosen for studying the effects of temperature and $\mathrm{pH}$ on the vibrational spectra of NS hydrogels. As a matter of fact, the tuning of water content of hydrogels below the critical hydration level $h=5$ ensures that the system is far from saturation of the water confinement sites, indeed occurring at higher hydration levels.

\section{B Temperature-evolution of FTIR-ATR spectra}

Fig. 3(a) and (b) show the temperature-evolution of the FTIRATR spectra collected for the NS polymer hydrated at $h=4$ $(\mathrm{pH}=8.9)$ in two distinct spectral regions, i.e. $2700-3900$ and 1400-1800 $\mathrm{cm}^{-1}$. In these specific wavenumber windows, the characteristic intra-molecular $\mathrm{OH}$ stretching band and the $\mathrm{HOH}$ bending mode of water molecules engaged in the polymer network can be detected.

As already mentioned, ${ }^{36-39}$ these vibrational features of water are very sensitive to the different $\mathrm{H}$-bonding environments developed by $\mathrm{H}_{2} \mathrm{O}$ molecules ${ }^{43}$ in the bulk and the confined state and they appear more intense in the IR spectra than in Raman spectra.

The spectral modifications exhibited by the profiles of Fig. 3(a) with increasing temperature are consistent with the trend observed for the $\mathrm{OH}$ stretching mode of bulk water and widely reported in the literature. ${ }^{38,40,41}$ In particular, the shift towards higher wavenumbers and the corresponding reduction of the low-frequency shoulder observed for the broad profile of the $\mathrm{OH}$ stretching band have been related to the decrease of the population of ordered tetrahedral $\mathrm{H}$-bonded structures of $\mathrm{H}_{2} \mathrm{O}$ molecules, due to the thermal motion. ${ }^{36,38}$ At the same time, the temperature-evolution of the $\mathrm{HOH}$ bending mode of water can be followed in Fig. 3(b). Although this vibration (falling at about $1640 \mathrm{~cm}^{-1}$ ) appears convoluted in the spectra of hydrogels with the signals arising from the NS polymer, a red shift and peak sharpening with increasing temperature can be recognized in the IR spectra. The temperature response is thus opposite to that shown by the $\mathrm{OH}$ stretching band of water, and it has been observed in bulk water, ${ }^{38}$ in the case of aqueous

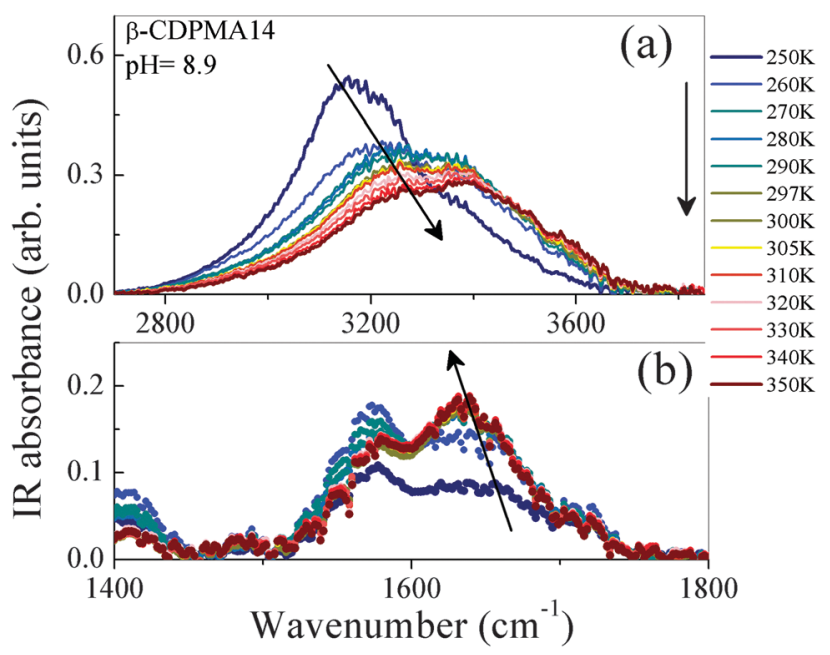

Fig. 3 Temperature-evolution of the infrared spectra obtained for the $\beta$-CDPMA14 nanosponge hydrated with $\mathrm{H}_{2} \mathrm{O}+\mathrm{Na}_{2} \mathrm{CO}_{3} 10 \% \mathrm{w} / \mathrm{w}(\mathrm{pH}=8.9)$ at $h=4$ in the spectral ranges (a) $2700-3900 \mathrm{~cm}^{-1}$ and (b) $1400-1800 \mathrm{~cm}^{-1}$. The arrows indicate the increase of temperature. 
solutions of sugars $^{16}$ and in water confined in zeolitic frameworks. ${ }^{6}$

\section{Analysis of $\mathrm{HOH}$ bending mode of water}

The temperature-dependence of $\mathrm{HOH}$ bending mode of water was carefully analysed by isolating such mode from the total IR experimental profile of NS hydrogels. The isolation of the water bending mode can be achieved by a strategy already successfully applied on similar gel systems and detailed in ref. 22 .

Fig. 4(a) compares the IR spectra collected on samples of the NS polymer hydrated with water and deuterated water at the same hydration level $(h=4)$ and under the same $\mathrm{pH}$ conditions. As expected, the spectra of hydrogel prepared with $\mathrm{D}_{2} \mathrm{O}$ exactly reproduce the vibrational signals arising from the hydrated polymer matrix but without the presence of the $\mathrm{HOH}$ bending mode of engaged water molecules.

This suggests us to use the spectra of NSs hydrated with $\mathrm{D}_{2} \mathrm{O}$ for obtaining a reliable estimate of the vibrational contribution associated with the polymer matrix in the complex spectra of hydrogels. Starting from the fitting of the $\mathrm{D}_{2} \mathrm{O}$-hydrogel spectra, ${ }^{22}$ the vibrational modes of NSs, falling in the spectral region 1500$1800 \mathrm{~cm}^{-1}$, have been modelled as a sum of four Voigt functions. ${ }^{22,44}$ The spectral components found in this way for the NS modes (grey colored area in Fig. 4(b)) have been then subtracted from the experimental IR spectra of NSs hydrated with $\mathrm{H}_{2} \mathrm{O}$, providing the so-called NS-free spectrum. This spectral profile contains only the vibrational contribution arising from the water molecules confined in the pores of the NS hydrogel matrix. The panels of Fig. 4(b) display an example of NS-free spectra obtained by following the strategy described above for NS hydrogels at two different temperatures, where the vibrational feature corresponding to the $\mathrm{HOH}$ bending mode of water is evident.

The temperature-evolution of the $\mathrm{HOH}$ bending mode isolated in the NS-free spectra is shown in Fig. 4(c). From inspection of the curves, it firstly appears that the $\mathrm{HOH}$ bending mode of water at $250 \mathrm{~K}$ shows a characteristic Gaussian-like shape different from the nearly flattened profile typically exhibited by polycrystalline ice at the same temperature. ${ }^{8,38,45}$ This suggests the presence of $\mathrm{H}_{2} \mathrm{O}$ molecules confined in the polymer network of NSs that are in a liquid-like state even below the nucleation temperature where usually the bulk water crystallizes. ${ }^{38,45}$ This behaviour, recalling the trend found for the water molecules confined in different kinds of nano-porous organic and inorganic systems, ${ }^{8,22,46,47}$ is consistent with the conclusion that water can be strongly confined in the nano-cavities of the polymer network of this type of $\mathrm{pH}$-sensitive NS hydrogel.

Fig. 4(c) also points out that the temperature-evolution of $\mathrm{HOH}$ bending mode of water in NS hydrogels follows the same red-shift and an increase of intensity observed for bulk water. ${ }^{38}$ This behaviour has been interpreted in terms of reduced population of ordered tetrahedral $\mathrm{H}$-bonded structures of water molecules due to the thermal motion, ${ }^{38}$ and it is in agreement

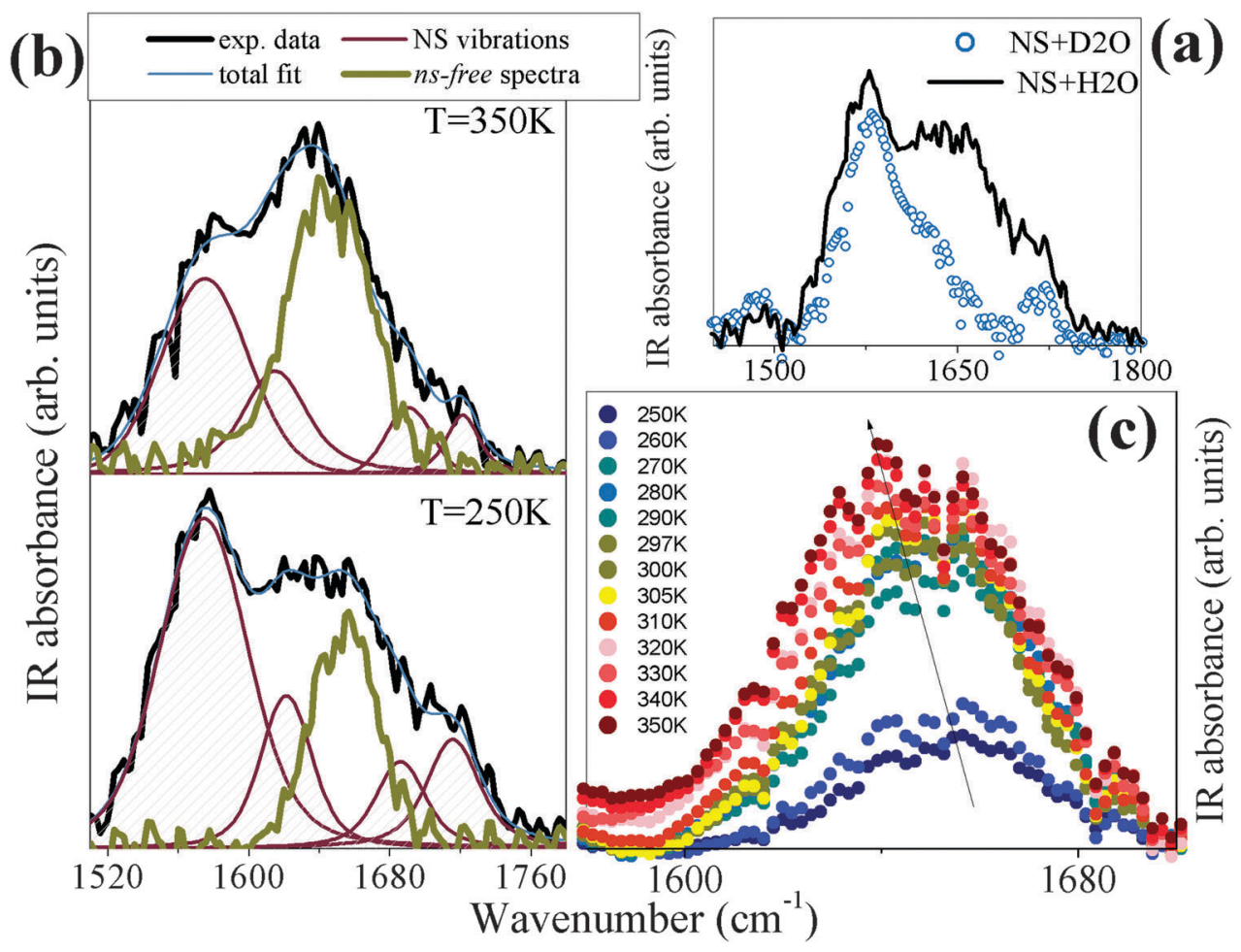

Fig. 4 (a) Infrared spectra obtained for the $\beta$-CDPMA14 nanosponge hydrogel hydrated in $\mathrm{H}_{2} \mathrm{O}+\mathrm{Na}_{2} \mathrm{CO}_{3} 10 \% \mathrm{w} / \mathrm{w}$ (line) and $\mathrm{D}_{2} \mathrm{O}+\mathrm{Na} \mathrm{CO}_{3} 10 \% \mathrm{w} / \mathrm{w}$ (symbols) at $250 \mathrm{~K}$. (b) An example of the best fit for $\beta$-CDPMA14 hydrated with $\mathrm{H}_{2} \mathrm{O}+\mathrm{Na}_{2} \mathrm{CO}_{3} 10 \% \mathrm{w} / \mathrm{w}$ at $h=4$ and two different temperatures. The NS-free spectra (yellow lines) are obtained by subtracting the vibrational contribution of nanosponges (grey colored area) estimated from the multiple Voigt function curve fitting procedure (see text for details). (c) Temperature-evolution of NS-free spectra obtained for the $\beta$-CDPMA14 nanosponge hydrated with $\mathrm{H}_{2} \mathrm{O}+\mathrm{Na}_{2} \mathrm{CO}_{3} 10 \% \mathrm{w} / \mathrm{w}(\mathrm{pH}=8.9)$ at $h=4$ in the spectral range $1600-1700 \mathrm{~cm}^{-1}$. The arrow indicates the increase of temperature. 


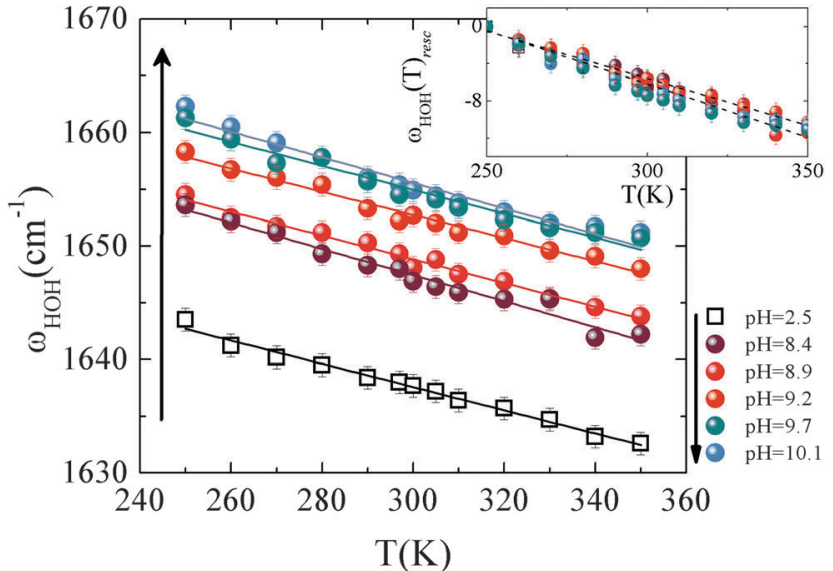

Fig. 5 Temperature-dependence of the frequency position of the $\mathrm{HOH}$ bending mode of water $\omega_{\mathrm{HOH}}$ obtained for the $\beta$-CDPMA14 nanosponge hydrogel hydrated at different $\mathrm{pH}$ values. Continuous lines are linear fit of the data. The arrows indicate the increase of $\mathrm{pH}$ measured in the hydrogel. Inset: frequency position $\omega_{\mathrm{HOH}}(T)$ rescaled for $\omega_{\mathrm{HOH}}(T=250 \mathrm{~K})$ as described in the text. The dashed lines represent the minimum and maximum slope of the data.

with the trend already discussed for the $\mathrm{OH}$ stretching mode of water in hydrogels (see Fig. 3(a)).

The distribution of different water species in NS hydrogels with increasing temperature can be conveniently studied by monitoring the frequency position of the $\mathrm{HOH}$ bending mode of water $\omega_{\mathrm{HOH}}$. This descriptor can be used for the quantitative evaluation of the destructuring effects induced by thermal motion on the HB network of water molecules.

Fig. 5 shows the temperature-dependence of the frequency position $\omega_{\mathrm{HOH}}$ extracted by the analysis of NS-free spectra obtained for NS polymers hydrated under different $\mathrm{pH}$ conditions.

The data reported in Fig. 5 show a linear relationship between $\omega_{\mathrm{HOH}}$ and $T$, for all of the $\mathrm{pH}$ values considered. Interestingly, the slope of the plots reported in Fig. 5 does not depend on the $\mathrm{pH}$ values. This appears evident by inspecting the temperature-decrease of the rescaled frequency position $\omega_{\mathrm{HOH}}(T)_{\text {resc }}$ (inset of Fig. 5), obtained as:

$$
\omega_{\mathrm{HOH}}(T)_{\mathrm{resc}}=\omega_{\mathrm{HOH}}(T)-\omega_{\mathrm{HOH}}(T=250 \mathrm{~K})
$$

By rescaling the position of bending mode for the value of frequency $\omega_{\mathrm{HOH}}$ measured at $250 \mathrm{~K}$, the data merge in a single linear master curve, as shown in the inset of Fig. 5. This finding leads to the conclusion that the extent of destructuring effects on the HB pattern of confined water molecules, induced by thermal motion, is not affected by the $\mathrm{pH}$. This behaviour is opposite to that brought about by the change in the crosslinking degree of the polymer network observed for similar NS hydrogels. ${ }^{22}$ In the latter case, the slope of the linear regression of $\omega_{\mathrm{HOH}} v s . T$ showed a clear dependence on the cross-linking degree of the polymer network, with the maximum value observed for the highest reticulated NS.

The linear regression lines of Fig. 5 suggest a constant, $\mathrm{pH}$ independent entropic contribution characterizing the HB rearrangement of water molecules inside the gel network of nanosponges.
From the experimental viewpoint, this confirms that the spectral features of $\mathrm{HOH}$ bending mode of water in these systems are closely and selectively connected to the properties of water confined in the polymeric backbone, thus representing an important investigation tool so far seldom exploited.

\section{Effect of $\mathrm{pH}$ on $\mathrm{HB}$ reorganization of confined water}

The linear plots reported in Fig. 5 display an increasing value of the intercept as a function of $\mathrm{pH}$ in the gel phase, indicating that the fraction of ordered (tetrahedral) water species present in the NS hydrogel network tends to grow at the expense of the "closed structures" of $\mathrm{H}_{2} \mathrm{O}$ molecules at high $\mathrm{pH}$ values.

The superimposition of the NS-free spectra obtained at $250 \mathrm{~K}$ for the NS polymer hydrated at different $\mathrm{pH}$ values is reported in Fig. 6.

The graph also shows the blue-shift and the intensity decrease of the $\mathrm{HOH}$ bending mode of water confined in NS hydrogels with increasing $\mathrm{pH}$, recalling the effect induced by cooling of the system on the vibrational features of water. To the best of our knowledge, this $\mathrm{pH}$-temperature superposition effect is unprecedented. Interestingly, the cooling effect (i.e. blue-shift of $\omega_{\mathrm{HOH}}$ ) carried out by $\mathrm{pH}$ changes is observable especially at basic $\mathrm{pH}$ (see inset of Fig. 6). A clear-cut interpretation of the $\mathrm{pH}$-induced blue-shift can be hardly proposed at this stage, however the important issue of modulation of the hydrophobic/hydrophilic ratio in the polymer backbone should be addressed. In particular, the following points should be kept in mind to find a rationale: (i) the hydrophobic/hydrophilic balance in the polymer backbone is affected by the $\mathrm{pH}$ of the medium due to the change in the ionization degree of the $\mathrm{COOH}$ groups in the polymer. Moving towards basic conditions, the dangling $\mathrm{COOH}$ groups originated by the polymerization of pyromellitic anhydride with cyclodextrin progressively turn into the anionic form. The literature $\mathrm{p} K_{\mathrm{a}}$ values of pyromellitic acid -1.8 ,

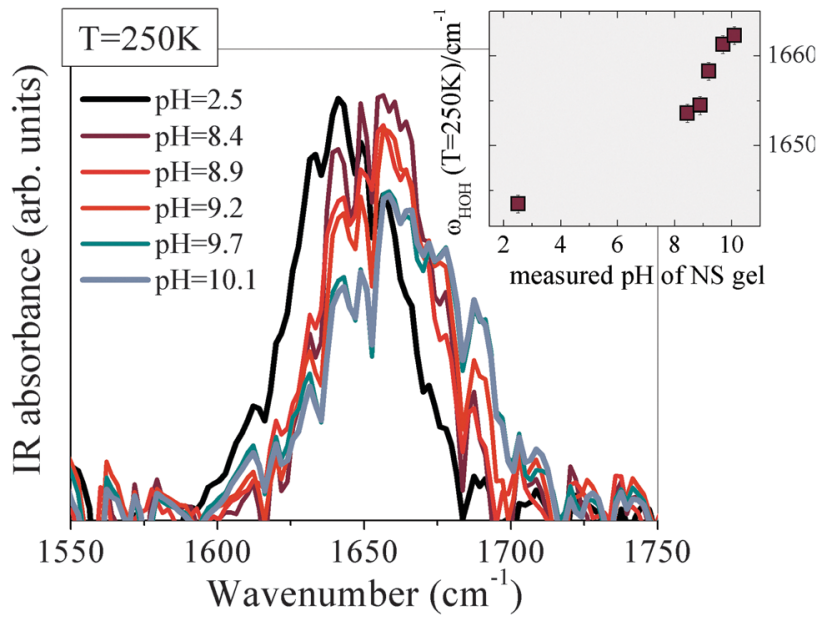

Fig. 6 NS-free spectra, reported in the spectral range $1550-1750 \mathrm{~cm}^{-1}$, obtained at $250 \mathrm{~K}$ for the $\beta$-CDPMA14 nanosponge hydrated under different $\mathrm{pH}$ conditions. Inset: Frequency position of the bending mode $\omega_{\mathrm{HOH}}$ estimated at $250 \mathrm{~K}$ as a function of $\mathrm{pH}$ measured in the $\beta$-CDPMA14 nanosponge hydrogel. 
2.8, 4.5 and 5.8, in the order - confirm this conclusion. (ii) In a parallel way, the hydrophobicity of the aromatic parts of the polymer is reduced with increasing $\mathrm{pH}$. Indeed, we have recently reported that the accessibility of water to the aromatic ring of the cross-linker in PMA based nanosponges grows on passing from the acid to the alkaline conditions. This conclusion was based on the analysis of the vibrational dephasing time $\left(\tau_{\mathrm{deph}}\right)$ associated with the $\nu(\mathrm{C}=\mathrm{C})_{1}$ vibration mode ${ }^{24}$ (see Section A of Results and discussion). The two factors discussed above point towards the enhancement of the hydrophilic characteristic of the nanosponge, and are related to the replacement of "closed structures" of $\mathrm{H}_{2} \mathrm{O}$ molecules by ordered (tetrahedral) water species.

The change in the ionization state of the $\mathrm{COOH}$ groups has another important consequence. As stated before, higher $\mathrm{pH}$ values result in higher frequency of the water bending mode $\omega_{\mathrm{HOH}}$. This finding mirrors the cooling-effect on the same vibrational feature observed for $\mathrm{H}_{2} \mathrm{O}$ adsorbed onto zeolitic frameworks. In such a case it underlined a relationship between $\omega_{\mathrm{HOH}}$ and the size of the pores where the water molecules were confined in the solid matrix. The population of water molecules involved in the extended, transient HB with a high degree of connectivity was proved to increase, even upon confinement, as a consequence of the enlargement of the nanopores of the confining matrix. ${ }^{6}$ Accordingly, the data in Fig. 6 suggest that high $\mathrm{pH}$ values in NS hydrogels lead to a possible enlargement of the hydrogel network mesh size, ${ }^{48}$ thus favouring the enhancement of the swelling capability of the system. ${ }^{24,26} \mathrm{We}$ are currently checking this hypothesis by small angle neutron scattering measurements at variable $\mathrm{pH}$ values. This behaviour is likely to be related to the deprotonation of carboxylic groups of the NS polymer under basic conditions, which causes the electrostatic repulsion of negative charges in the network and thus the increase of the pore volume available for the water molecules during the swelling process. ${ }^{48}$

This conclusion seems to establish a link between the molecular effects of the pore enlargement in NS hydrogels that can be efficiently controlled by varying the $\mathrm{pH}$ of hydration, and the macroscopic properties of swelling exhibited by the polymer.

\section{Conclusions}

This paper deals with the case study of water confined in the hydrophilic cavities of $\mathrm{pH}$-sensitive cyclodextrin nanosponge (NS) hydrogels. The vibrational signatures of the reorganization of the HB network of water molecules and the solvation-effects on hydrophobic/hydrophilic groups of the polymer backbone in the hydrogel state are disentangled and selectively followed at variable temperatures, hydration levels and $\mathrm{pH}$ of the system. In the case of pH-sensitive NS hydrogels, the solvation effects are essentially dominated by the hydrophobic hydration of the more hydrophobic parts of the NS structure. This outcome suggests the importance of regulating the hydrophobic/hydrophilic balance of chemical groups in the polymer network to achieve a suitable tuning of the solvation properties in the NS hydrogel. Secondarily, the detailed analysis of $\mathrm{HOH}$ bending mode of water evidences a clear influence of $\mathrm{pH}$ on the $\mathrm{HB}$ reorganization of confined water molecules that tend to develop an increasing number of ordered tetrahedral structures, even upon confinement, at high $\mathrm{pH}$ values. A possible rationale is related to two different factors: the enhancement of hydrophilic components of the polymeric backbone and the enlargement of pores in NS hydrogels, occurring under basic conditions and leading to the enhancement of the swelling ability observed at a macroscopic level.

\section{Acknowledgements}

Regione Toscana (POR FESR 2014-2020, Call RSI 2014, and Project NanoBonD) is acknowledged for financial support.

\section{Notes and references}

1 M. C. Bellissent-Funel, Eur. Phys. J. E: Soft Matter Biol. Phys., 2003, 12, 83.

2 T. Tsukahara, A. Hibara, Y. Ikeda and T. Kitamori, Angew. Chem., Int. Ed., 2007, 46, 1180.

3 S. Le Caer, S. Pin, S. Esnouf, Q. Raffy, J. Ph. Renault, J. B. Brubach, G. Creff and P. Roy, Phys. Chem. Chem. Phys., 2011, 13, 17658.

4 J. B. Brubach, A. Mermet, A. Filabozzi, A. Gerschel, D. Lairez, M. P. Krafft and P. Roy, J. Phys. Chem. B, 2001, 105, 430.

5 J. Dore, Chem. Phys., 2000, 258, 327.

6 V. Crupi, F. Longo, D. Majolino and V. Venuti, J. Phys.: Condens. Matter, 2006, 18, 3563.

7 V. Crupi, S. Interdonato, F. Longo, D. Majolino, P. Migliardo and V. Venuti, J. Raman Spectrosc., 2008, 39, 244.

8 F. Mallamace, M. Broccio, C. Corsaro, A. Faraone, D. Majolino, V. Venuti, L. Liu, C. Y. Mou and S. H. Chen, Proc. Natl. Acad. Sci. U. S. A., 2007, 104, 424.

9 J. Teixeira, J. M. Zanotti, M.-C. Bellissent-Funel and S.-H. Chen, Physica B, 1997, 234-236, 370.

10 L. Liu, S. H. Chen, A. Faraone, C. W. Yen and C. Y. Mou, Phys. Rev. Lett., 2005, 95, 117802.

11 P. C. Shih, H. P. Lin and C. Y. Mou, Stud. Surf. Sci. Catal., 2003, 146, 557.

12 F. Mallamace, M. Broccio, C. Corsaro, A. Faraone, U. Wanderlingh, L. Liu, C. Y. Mou and S. H. Chen, J. Chem. Phys., 2006, 124, 161102.

13 D. Laage and J. Hynes, Science, 2010, 311, 832.

14 R. A. Nicodemus, K. Ramasesha, S. T. Roberts and A. Tokmakoff, J. Phys. Chem. Lett., 2010, 1, 1068.

15 B. Bagchi, Chem. Rev., 2005, 105, 3197.

16 M. E. Gallina, P. Sassi, M. Paolantoni, A. Morresi and R. S. Cataliotti, J. Phys. Chem. B, 2006, 110, 8856.

17 S. Perticaroli, L. Comez, M. Paolantoni, P. Sassi, A. Morresi and D. Fioretto, J. Am. Chem. Soc., 2011, 133, 12063.

18 D. Russo, J. Ollivier and J. Teixeira, Phys. Chem. Chem. Phys., 2008, 10, 4968.

19 L. Comez, L. Lupi, A. Morresi, M. Paolantoni, P. Sassi and D. Fioretto, J. Phys. Chem. Lett., 2013, 4, 1188. 
20 K. Wood, M. Plazanet, F. Gabel, B. Kessler, D. Oesterhelt, D. J. Tobias, G. Zaccai and M. Weik, Proc. Natl. Acad. Sci. U. S. A., 2007, 104, 18049.

21 B. Rossi, V. Venuti, F. D’Amico, A. Gessini, F. Castiglione, A. Mele, C. Punta, L. Melone, V. Crupi, D. Majolino, F. Trotta and C. Masciovecchio, Phys. Chem. Chem. Phys., 2015, 17, 963.

22 B. Rossi, V. Venuti, A. Mele, C. Punta, L. Melone, V. Crupi, D. Majolino, F. Trotta, F. D’Amico, A. Gessini and C. Masciovecchio, J. Chem. Phys., 2015, 142(1), 014901.

23 V. Venuti, B. Rossi, F. D’Amico, A. Mele, C. Punta, L. Melone, V. Crupi, D. Majolino, F. Trotta, A. Gessini and C. Masciovecchio, Phys. Chem. Chem. Phys., 2015, 17, 10274.

24 B. Rossi, V. Venuti, F. D’Amico, A. Gessini, A. Mele, C. Punta, L. Melone, V. Crupi, D. Majolino, F. Trotta and C. Masciovecchio, Soft Matter, 2015, 11, 5862.

25 F. Trotta, M. Zanetti and R. Cavalli, Beilstein J. Org. Chem., 2012, 8, 2091.

26 M. Ferro, F. Castiglione, C. Punta, L. Melone, W. Panzeri, B. Rossi, F. Trotta and A. Mele, Beilstein J. Org. Chem., 2014, 10, 2715.

27 V. Crupi D. Majolino, A. Mele, L. Melone, C. Punta, B. Rossi, F. Toraldo, F. Trotta and V. Venuti, Soft Matter, 2014, 10, 2320.

28 S. Subramanian, A. Singireddy, K. Krishnamoorthy and M. Rajappan, J. Pharm. Pharm. Sci., 2012, 15(1), 103.

29 S. V. Chilajwar, P. P. Pednekar, K. R. Jadhav, G. J. C. Gupta and V. J. Kadam, Expert Opin. Drug Delivery, 2014, 11(1), 111.

30 B. Rossi, S. Caponi, F. Castiglione, S. Corezzi, A. Fontana, M. Giarola, G. Mariotto, A. Mele, C. Petrillo, F. Trotta and G. Viliani, J. Phys. Chem. B, 2012, 116(17), 5323.

31 B. Rossi, V. Venuti, A. Paciaroni, A. Mele, S. Longeville, F. Natali, V. Crupi, D. Majolino and F. Trotta, Soft Matter, 2015, 11, 2183.
32 V. Crupi, A. Fontana, M. Giarola, S. Longeville, D. Majolino, G. Mariotto, A. Mele, A. Paciaroni, B. Rossi, F. Trotta and V. Venuti, J. Phys. Chem. B, 2014, 118(2), 624.

33 F. Castiglione, V. Crupi, D. Majolino, A. Mele, B. Rossi, F. Trotta and V. Venuti, J. Phys. Chem. B, 2012, 116(43), 13133. 34 F. Castiglione, V. Crupi, D. Majolino, A. Mele, B. Rossi, F. Trotta and V. Venuti, J. Phys. Chem. B, 2012, 116(27), 7952. 35 G. W. Robinson, C. H. Cho and J. Urquidi, J. Chem. Phys., 1999, 111, 698.

36 G. E. Walrafen, M. S. Hokmababi and W. H. Yang, J. Chem. Phys., 1986, 85, 6964.

37 G. D’Arrigo, G. Maisano, F. Mallamace, P. Migliardo and F. Wanderlingh, J. Chem. Phys., 1981, 75, 4264.

38 B. Brubach, A. Mermet, A. Filabozzi, A. Gerschel and P. Roy, J. Chem. Phys., 2005, 122, 184509.

39 M. Paolantoni, N. Faginas lago, M. Albert and A. Lagana, J. Phys. Chem. A, 2009, 113, 15100.

40 F. O. Libnau, J. Toft, A. A. Christy and O. M. Kvalheim, J. Am. Chem. Soc., 1994, 116, 8311.

41 Y. Marechal, J. Chem. Phys., 1991, 95(8), 5565.

42 F. D’Amico, M. Saito, F. Bencivenga, M. Marsi, A. Gessini, G. Camisasca, E. Principi, R. Cucini, S. DiFonzo and A. Battistoni, Nucl. Instrum. Methods Phys. Res., Sect. A, 2013, 703, 33.

43 G. E. J. Walrafen, Chem. Phys., 1967, 47, 19114.

44 V. Crupi, D. Majolino, A. Mele, B. Rossi, F. Trotta and V. Venuti, Soft Matter, 2013, 9, 6457.

45 A. Millo, Y. Raichlin and A. Katzir, Appl. Spectrosc., 2005, 59, 460. 46 V. Crupi, D. Majolino, P. Migliardo, V. Venuti and M. C. Bellissent-Funel, Mol. Phys., 2003, 101, 3323.

47 V. Crupi, F. Longo, D. Majolino and V. Venuti, J. Chem. Phys., 2005, 123, 154702.

48 N. A. Peppas, J. Z. Hilt, A. Khademhosseini and R. Langer, Adv. Mater., 2006, 18, 1345. 